\title{
Long-term Effects of Micronized Alloderm Injection for Unilateral Vocal Fold Paralysis
}

Claudio F. Milstein, PhD; Lee M. Akst, MD; M. Douglas Hicks, PhD; Tom I. Abelson, MD; Marshall Strome, MD

Objectives: Micronized Alloderm (Cymetra) is a relatively new product used for vocal fold augmentation. Previous studies evaluating possible long-term effectiveness of this product have shown mixed results. The objective of this present study is to reassess possible long-term results of Cymetra injection laryngoplasty in patients with unilateral true vocal fold paralysis. Study Design: Retrospective review of patients with unilateral true vocal fold paralysis who received Cymetra injection laryngoplasty between March 2001 and March 2004. Methods: Preoperative voice samples and videostroboscopic findings were compared with the most recently available postoperative data to assess efficacy of the procedure. A panel of voice experts analyzed both vocal and vibratory function in these samples. In addition, pre- and postoperative voice-related quality of life measures and patients' self-ratings of voice outcomes were compared. Results: Twenty patients ( 7 male, 13 female; 14 with left-sided paralysis, 6 with right-sided paralysis) were identified in the study population. Cymetra injection was performed an average of 45.1 months after onset of vocal fold paralysis (range -216 months), and average follow-up postinjection was 11.2 (range -35) months. Comparing pre- and postoperative measures, voice quality $(P<.0001)$, glottal closure $(P<.0001)$, and degree of vocal fold bowing $(P<.0001)$ were all improved by injection. Quality of life measures and patients' self-perceptions of vocal quality were also improved $(P<.01)$. Fifteen $(75 \%)$ patients showed longlasting results. Eight patients showed improvement for more than 12 months after injection. Conclusion: Cymetra injection laryngoplasty offers improved vocal and vibratory function to patients with unilateral true vocal fold paralysis. The benefits of such medialization may be longer lasting than previously reported, and further long-term study is warranted. Key Words: Vocal fold paralysis, injection laryngoplasty, vocal fold medialization, voice quality, quality of life.

Laryngoscope, 115:1691-1696, 2005

From the Cleveland Clinic Foundation, Cleveland, Ohio, U.S.A. 2005.

Editor's Note: This Manuscript was accepted for publication May 24,

Send Correspondence to Dr. Claudio F. Milstein, The Voice Center, Head and Neck Institute, 9500 Euclid Avenue A71, Cleveland, Ohio 44195, U.S.A.

DOI: $10.1097 / 01 . m l g .0000173163 .07828 .30$

\section{INTRODUCTION}

Unilateral true vocal fold paralysis is a common problem encountered in an otolaryngology clinic. The symptoms of dysphonia, dysphagia, and even aspiration, which may accompany unilateral vocal fold paralysis, can be very disturbing to patients. ${ }^{1}$ Fortunately, there are several techniques that can address these issues, including among them voice therapy, injection laryngoplasty, medialization, and laryngeal reinnervation procedures. Much has been written about each of these procedures, and there are advantages and disadvantages to each.

Since its initial description by Brunings ${ }^{2}$ in 1911 and subsequent reintroduction by Arnold ${ }^{3}$ in the 1960s, injection laryngoplasty has become popular because of its immediate benefit and relatively noninvasive nature. The difficulty with injection laryngoplasty comes with finding an ideal material for injection. Hypothetically, the ideal injectable should be biologically well tolerated, easily injected, and resistant to resorption after injection. ${ }^{3}$ In addition, the ideal injectable should not interfere with the pliability of true vocal fold mucosal vibration, should be easily reversible (explantable), and should not migrate from the site of injection. ${ }^{4}$

Regrettably, no material as yet meets all of these criteria as an "ideal" bio-injectable. Teflon may cause inflammation, granuloma formation, compromise of mucosal waves, and may extrude. ${ }^{5,6}$ Gelfoam is quickly absorbed and offers only temporary rehabilitation. ${ }^{7}$ Autologous fat and bovine collage injection are subject to unpredictable resorption with variable voice outcomes. ${ }^{8,9}$ Autologous collagen involves donor-site morbidity and requires processing time between harvest and injection. ${ }^{10}$

A newer injectable material AlloDerm (LifeCell Corporation, Branchburg, NJ) is a nonimmunogenic, acellular dermal matrix that is derived from processed cadaveric dermis; the micronized form of AlloDerm (Cymetra, LifeCell Corporation) preserves the acellular collagen and elastin matrix of the original in an injectable form. ${ }^{4}$ This material has been used for soft tissue augmentation since the early 1990s, with no reports of safety complications or disease transmission.11-14 It has shown good biocompatibility and volume persistence at 1 and 3 month time points. ${ }^{11}$ However, the results of Cymetra injection for treatment of unilateral true vocal fold paralysis have been mixed, particularly with re- 
gard to possible long-term effects.,11,16 Therefore, we decided to retrospectively review patients with unilateral vocal fold paralysis who underwent Cymetra injection laryngoplasty at our institution to determine long-term efficacy and voice quality.

\section{METHODS}

A retrospective review was conducted to identify all patients who underwent Cymetra injection laryngoplasty performed by the senior author (M.S.) during the 3 year period from March 2001 through March 2004. Data collection was approved by the Institutional Review Board of the Cleveland Clinic Foundation. Patients were chosen for study inclusion on the basis of known unilateral true vocal fold paralysis, which was symptomatic relative to either dysphonia or dysphagia.

Injection laryngoplasty was performed in each case in the operating room, with the patient under general anesthesia. Injection was performed with suspension microlaryngoscopy. Patients were either intubated with small ( $5 \mathrm{~mm}$ inner diameter) endotracheal tubes or underwent Venturi "jet" ventilation for the duration of the case. Preoperative photographs of the larynx were available at the time of injection to guide location and amount of injection. Cymetra was formulated according to the instructions provided by the manufacturer (LifeCell Corporation) and then injected through a Xomed laryngeal injection needle with a disposable 27 gauge needle. The first injection was performed immediately lateral to the vocal process, with further injections performed 2 to $3 \mathrm{~mm}$ lateral to the vocal ligament at the midcordal level. In anticipation of some resorption, each cord was over-injected by approximately $10 \%$.

For data collection, preoperative digital voice samples and videostroboscopy were compared with the most recently available postoperative voice sample and videostroboscopy. A panel of three voice experts, all experienced in the analysis of videostroboscopy, then assessed vocal fold bowing, glottal competence, and voice quality on a 1 to 4 point scale $(1=$ best, $4=$ worst). These ratings were performed in a blinded fashion, with the observers unaware of patient identity or pre- versus postoperative status at the time of ratings.

In addition to these third-party ratings, the efficacy of injection laryngoplasty was also assessed through patient perception of vocal performance. Here, patients were asked to rate their voice quality both before and after surgery on a 1 (worst) to 10 (best, "normal voice") scale. In addition, patients filled out a validated voice-related quality of life instrument (V-RQOL, Hogikyan and Sethuraman $)^{15}$ for both their pre- and postoperative voices. Last, patients were asked after surgery to rate their degree of voice change because of the procedure on an ordinal 5 point scale $(1=$ much worse, $2=$ somewhat worse, $3=$ no change, $4=$ somewhat better, $5=$ much better). For these self-measurements, patients filled out the appropriate forms both before and after surgery if surgery was performed after January 2002. For the three study patients who had surgery before January 2002, both pre- and postoperative perceptual data were obtained retrospectively.

Statistical analysis was then performed to assess the impact of the injection laryngoplasty on vocal function. All of the above measures, videostroboscopic appearance, experts' perceptual voice ratings, patients' self-perceived vocal quality scores, V-RQOL scores, and perceived degree of voice change, were analyzed, comparing preoperative with postoperative scores. Because of the relatively low number of subjects and the ordinal nature of the data, the Wilcoxon signed rank test was used to describe the change in scores at a $5 \%$ significance level.

Twenty patients were identified who received Cymetra injection laryngoplasty for unilateral true vocal fold paralysis from the senior author (M.S.) between March 2001 and March 2004. Patient demographics are displayed in Table I. More patients are female than male, and average age at time of treatment is $59.6 \pm 13.8$ years. Causes of vocal fold paralysis include 10 idiopathic or viral cases, 6 patients with cervical surgery or malignancy, and 4 patients with intrathoracic surgery or malignancy. Of note, the patients in this study display a wide range in the amount of time between onset of vocal fold paralysis and time of injection, ranging from 4 to 216 (mean $45 \pm 52$ ) months. For 16 of the20 patients, at least 12 months had passed between time of onset and time of injection, suggesting that further spontaneous recovery of vocal fold function was unlikely.

At the time of injection, patients received an average of 0.76 $\pm 0.59 \mathrm{~mL}$ of Cymetra (range $0.3-2.5 \mathrm{~mL}$, Table I; data not available for 3 patients). Patients were observed in the recovery room for several hours after injection, and no immediate airway complications were noted. In careful postoperative follow-up, there were also no documented instances of hematoma or infection after treatment. After injection, the most recent follow-up at the time of data analysis ranged from 1 to 35 months (mean 11.2 \pm 10.7 , Table I).

\section{RESULTS}

Overall results for both vocal and videostroboscopic measures are favorable when preoperative values are compared with postoperative results. Figure 1 shows a composition of videoendoscopic images before and after the procedure, depicting changes in configuration of glottal closure and degree of vocal fold bowing. A panel of trained experts judged the degree of glottal closure on a 1 to 4 scale, with higher scores reflecting higher degrees of glottal insufficiency ( 1 = complete closure; $2=$ mildly incomplete; $3=$ moderately incomplete; $4=$ severely incomplete) ( $\mathrm{n}=19$, data missing for one patient).

The percentage of patients with the highest scores for the glottal closure measure (3 and 4) decreased from $55 \%$ and $30 \%$, respectively, preoperatively, to $5 \%$ and $0 \%$ postoperatively. As a group, the mean scores changed from $3.21 \pm 0.63$ preoperatively to $1.42 \pm 1.33$ postoperatively $(P<.0001)$ (Table II) (Fig. 2$)$

As judged on a similar 4 point scale $(1=$ straight vocal fold edge; 2 = mildly bowed; $3=$ moderately bowed; $4=$ severely bowed $)(\mathrm{n}=19$, data missing for 1 patient), the degree of vocal fold bowing also significantly improved after injection. Fifty percent of patients were rated as moderately bowed and 5\% as severely bowed preoperatively. Both scores dropped to $0 \%$ postoperatively. Overall group mean scores changed from $2.38 \pm 0.92$ to $1.36 \pm$ $0.60(P<.0001)$ (Table II). Improved glottal closure and decreased vocal fold bowing were also manifested by decreased supraglottic compression and improved mucosal wave dynamics on videostroboscopy.

Vocal improvement was measured with a number of instruments, each of which demonstrates significant improvement after injection. Perceptual evaluation of voice quality was rated on a 1 to 4 point scale ( $1=$ normal voice; 2 = mild dysphonia; 3 = moderate dysphonia; $4=$ severe dysphonia). Moderate and severe dysphonia decreased from $50 \%$ and $35 \%$ preoperatively, to $20 \%$ and $0 \%$ postoperatively. Group mean scores reflected improvement in voice quality from $3.23 \pm 0.70$ before injection to $1.65 \pm$ 0.81 after injection $(P<.0001)$ (Table II) (Fig. 3). Patients' self-perceived voice qualities, meanwhile, improved on a 1 to 10 ( $1=$ severe dysphonia, $10=$ normal voice $)$ scale from $2.83 \pm 1.50$ to $7.89 \pm 1.84(P<.01)$. These raw indices are 
TABLE I.

Demographic Information.

\begin{tabular}{|c|c|c|c|c|c|c|c|}
\hline Patient & $\begin{array}{c}\text { Sex } \\
\text { (Male/Female) }\end{array}$ & $\begin{array}{l}\text { Age at } \\
\text { Treatment } \\
\text { (Yrs) }\end{array}$ & Etiology & $\begin{array}{c}\text { Side } \\
\text { (Left/Right) }\end{array}$ & $\begin{array}{l}\text { Time between } \\
\text { Onset and } \\
\text { Treatment (Mos) }\end{array}$ & $\begin{array}{l}\text { Amount } \\
\text { Injected } \\
(\mathrm{mL})\end{array}$ & $\begin{array}{l}\text { Follow-Up } \\
\text { (Mos) }\end{array}$ \\
\hline 1 & $\mathrm{~F}$ & 66 & Idiopathic & L & 13 & 0.5 & 35 \\
\hline 2 & $M$ & 75 & Thoracic & $\mathrm{R}$ & 6 & 0.6 & 33 \\
\hline 3 & $F$ & 85 & Idiopathic & L & 18 & 0.3 & 29 \\
\hline 4 & $\mathrm{~F}$ & 45 & Idiopathic & L & 119 & 2.5 & 22 \\
\hline 5 & $\mathrm{~F}$ & 52 & Viral & L & 48 & $\mathrm{n} / \mathrm{a}$ & 16 \\
\hline 6 & $M$ & 71 & Cervical & R & 4 & 2.0 & 12 \\
\hline 7 & $\mathrm{~F}$ & 42 & Idiopathic & L & 108 & 0.4 & 12 \\
\hline 8 & $F$ & 63 & Viral & L & 42 & 0.4 & 12 \\
\hline 9 & $M$ & 78 & Thoracic & L & 20 & 0.5 & 11 \\
\hline 10 & $\mathrm{~F}$ & 39 & Cervical & L & 38 & 1.0 & 8 \\
\hline 11 & $\mathrm{~F}$ & 71 & Thoracic & L & 54 & 0.5 & 8 \\
\hline 12 & $M$ & 63 & Cervical & $\mathrm{R}$ & 14 & $\mathrm{n} / \mathrm{a}$ & 7 \\
\hline 13 & $M$ & 55 & Thoracic & L & 11 & 0.5 & 4 \\
\hline 14 & $M$ & 78 & Idiopathic & L & 38 & 0.8 & 4 \\
\hline 15 & $F$ & 49 & Cervical & $\mathrm{R}$ & 84 & $\mathrm{n} / \mathrm{a}$ & 3 \\
\hline 16 & $M$ & 46 & Viral & L & 216 & 0.5 & 2 \\
\hline 17 & $\mathrm{~F}$ & 57 & Viral & L & 12 & 0.4 & 2 \\
\hline 18 & $\mathrm{~F}$ & 63 & Cervical & $\mathrm{R}$ & 4 & 0.8 & 1 \\
\hline 19 & $\mathrm{~F}$ & 41 & Cervical & $\mathrm{R}$ & 26 & 0.6 & 1 \\
\hline 20 & $\mathrm{~F}$ & 52 & Idiopathic & L & 27 & 0.7 & 1 \\
\hline Mean & & 59.6 & & & 45.1 & 0.76 & 11.2 \\
\hline SD & & 13.8 & & & 52.1 & 0.59 & 10.7 \\
\hline
\end{tabular}

corroborated by patients' own assessment of the benefits of vocal fold injection. When asked to report on a 5 point scale whether injection made their voice 1) "much worse," 2) "somewhat worse," 3) "unchanged," 4) "somewhat bet-

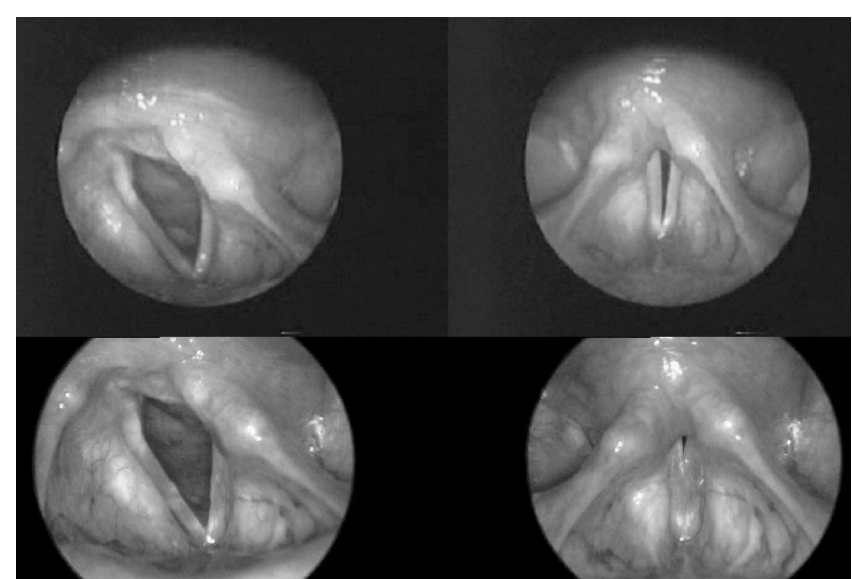

Fig. 1. Sample videoendoscopic images before and 6 months after the procedure. (Top left) Preoperative laryngeal image at rest. Note position of paralyzed left vocal fold (right of image) with mildly bowed edge. (Top right) Preoperative laryngeal image during phonation, showing the closed phase of the vibratory cycle, with a large glottal gap along the entire glottis. (Bottom left) 6-month postoperative laryngeal image of same patient. Note straight left vocal fold edge. (Bottom right) Six month postoperative laryngeal image during phonation, with much improved glottal closure. ter," or 5) "much better," patients overall judged that their posttreatment voices were much better than their pretreatment voices, with a mean score of $4.76 \pm 0.55$.

$\mathrm{V}$-RQOL scores provide one last measure of aggregate outcomes after injection laryngoplasty. In this measure, higher scores reflect higher V-RQOL. For the 14 patients from whom data are complete, V-RQOL scores improved from $33.4 \pm 20.4$ pretreatment to $78.0 \pm 16.4$ posttreatment $(P<.01)$. As shown in Figure 3, all of these patients experienced an improvement in V-RQOL, with no exceptions. Similar data hold true when the physical functional and social emotional domains are separated out from the total V-RQOL score (Figs. 4 and 5).

Although aggregate data analysis uses comparisons of preoperative voice and videostroboscopic evaluation as compared with the most recently available postoperative values, it is also interesting to examine what happened to individual patients after injection. For 5 of the 20 patients, the effects of injection were temporary. Three patients had initial benefit, which slowly decreased by 3 months postinjection; two patients experienced more dramatic resorption, with initial benefit followed by relatively quick return to pretreatment baselines. One patient of the latter group had a second injection, with repeated rapid resorption and return to baseline within 3 weeks of injection. The remaining 15 study patients have continued benefits from injection, with 8 of the 15 maintaining improvement for 1 year or longer after injection. 
TABLE II.

Pre- and Postoperative Findings in Measures of Vocal Function.

\begin{tabular}{|c|c|c|c|c|c|c|c|}
\hline & \multirow[b]{2}{*}{$\mathrm{n}$} & \multicolumn{4}{|c|}{ Score (\%) } & \multirow[b]{2}{*}{ Mean (SD) } & \multirow[b]{2}{*}{$P$ Value ${ }^{*}$} \\
\hline & & 1 & 2 & 3 & 4 & & \\
\hline \multicolumn{8}{|l|}{ Glottal closure } \\
\hline Pre-injection & 20 & $0(0)$ & $3(15)$ & $11(55)$ & $6(30)$ & $3.15(0.67)$ & \\
\hline Postinjection & 19 & $13(65)$ & $5(25)$ & $1(5)$ & $0(0)$ & $1.37(0.60)$ & $<.0001$ \\
\hline \multicolumn{8}{|l|}{ Vocal fold edge } \\
\hline Pre-injection & 20 & $5(25)$ & $4(20)$ & $10(50)$ & $1(5)$ & $2.35(0.93)$ & \\
\hline Postinjection & 19 & $13(65)$ & $6(30)$ & $0(0)$ & $0(0)$ & $1.32(0.48)$ & $<.0001$ \\
\hline \multicolumn{8}{|l|}{ Voice perception } \\
\hline Pre-injection & 20 & $0(0)$ & $3(15)$ & $10(50)$ & $7(35)$ & $3.2(0.70)$ & \\
\hline Postinjection & 20 & $11(55)$ & $5(25)$ & $4(20)$ & $0(0)$ & $1.65(0.81)$ & $<.0001$ \\
\hline
\end{tabular}

${ }^{*}$ The Wilcoxon signed rank test was used to determine whether the change from preoperative to postoperative is statistically significant.

When assessing long-term results, this study contains eight patients (patients 1-8) whose time of most recent follow-up was at least 12 months postprocedure. Analyzing this subset of patients with long-term follow-up reveals that as a group, the interval between onset of unilateral vocal fold paralysis and time of Cymetra injection is $44.8 \pm 45.4$ months, whereas average follow-up after injection is $21.4 \pm 9.8$ months. Some patients in this group have had substantial time pass between vocal fold paralysis and Cymetra injection: 13 months (patient 1), 18 months (patient 3), 42 months (patient 8), 48 months (patient 5), 108 months (patient 7), and 119 months (patient 4). In these patients, all of whom had benefits of Cymetra injection lasting at least 12 months, as documented by voice and videostroboscopic measures at the time of most recent follow-up, concomitant spontaneous recovery of vocal fold motion was unlikely.

\section{DISCUSSION}

As further investigation into injection laryngoplasty establishes its role in the treatment of unilateral vocal fold paralysis, the search for the ideal injection material continues. As seen by the overview provided in the introduction, any number of materials has been tested, but each has been found lacking in one or more areas of concern. Cymetra (micronized AlloDerm, Lifecell Corporation) has been one of the newest agents tried for injection laryngoplasty. ${ }^{4,11}$ As demonstrated in other models, it is biocompatible, ${ }^{11}$ and there is no donor-site morbidity associated with its use. In the vocal fold, past studies have shown this substance to provide good glottal closure at early time points; unfortunately, the reported vocal outcomes have been contradictory, and no long-term follow-up has been available. ${ }^{4,11,16}$ This study was performed to evaluate the vocal and videostroboscopic effects of Cymetra injection laryngoplasty using longer-term time points than previous studies.

Overall, the results of this study are favorable, with improvement in voice quality, glottal closure, and phonatory function. Similar to the previous studies by Pearl et al.,11 Karpenko et al., ${ }^{4}$ and Lundy et al., ${ }^{16}$ the current study finds that Cymetra closes glottal gaps in a statisti-

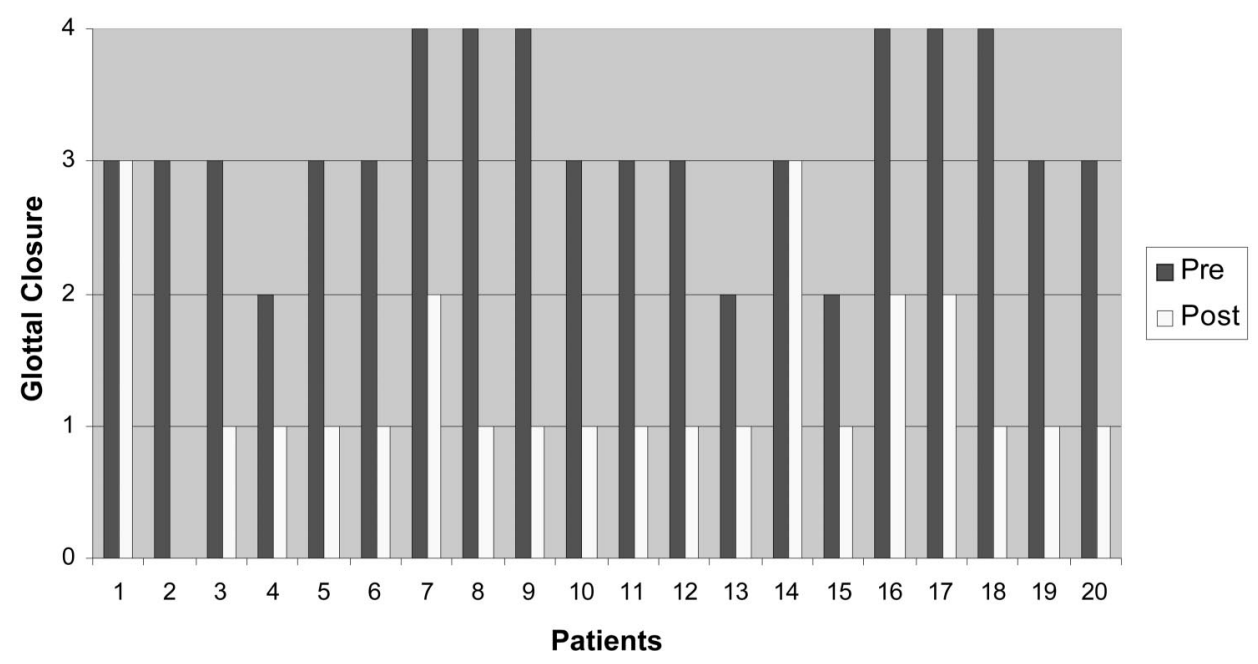

Fig. 2. Glottal closure ratings on a 4-point scale, comparing pre- and postoperative values for individual patients (missing postoperative data for patient 2). 
Fig. 3. Perceptual ratings of voice quality on a 4-point scale, comparing pre- and postoperativevalues for individual patients.

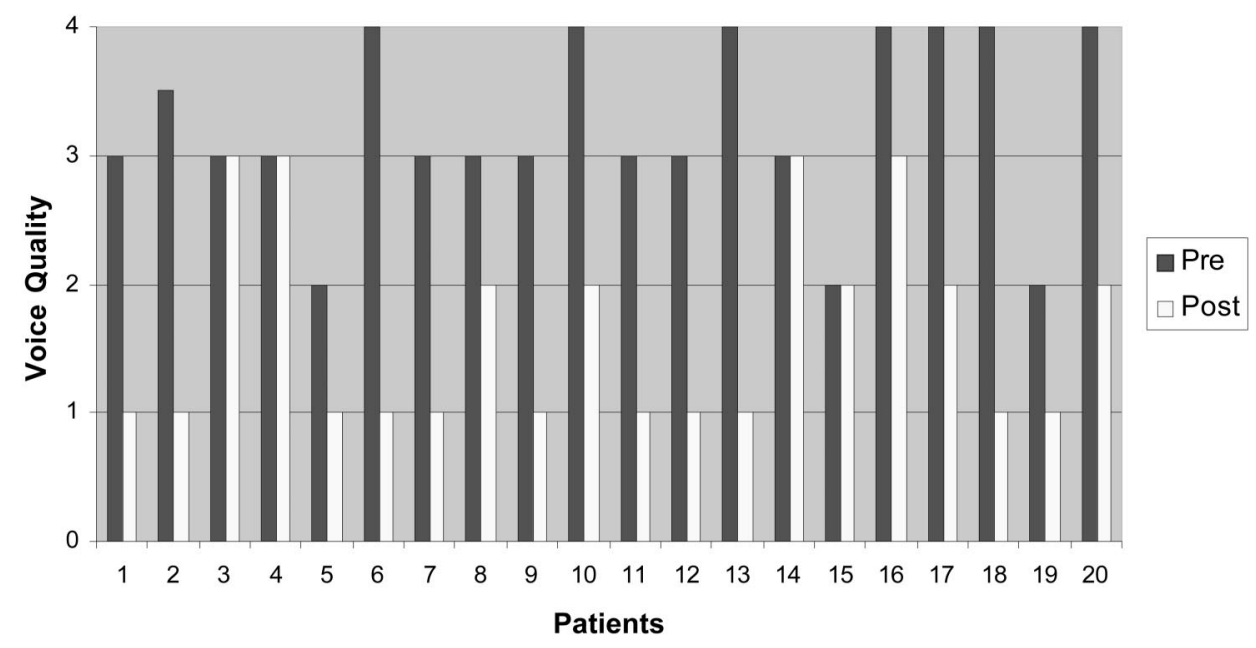

cally and clinically meaningful fashion. Furthermore, as also shown in the Pearl et al. study, the present study finds that restoration of glottal closure leads to an improved mucosal wave following injection; in other words, injection of this substance does not inhibit vibratory function. Importantly, these benefits are achieved without any significant complications. Similar to the studies mentioned above, this study finds Cymetra injection to be safe, without any episodes of airway compromise, hematoma, or infection. Of note, this study on average used less material $(0.76 \pm 0.59 \mathrm{~mL})$ than the $2.5 \mathrm{~mL}$ used by Zapanta and Bielamowicz ${ }^{17}$ in their description of a laryngeal abscess after injection laryngoplasty. The one patient in this study who did receive $2.5 \mathrm{~mL}$ of this material did so without complication.

Our findings showed statistical and clinical improvement in voice quality. On the basis of observers' ratings, patients' self ratings, patients' perceived voice change, and comparison of pretreatment and posttreatment V-RQOL, injection of this material improves voice in patients with unilateral vocal fold paralysis. Furthermore, this study suggests that injection may offer long-term results for greater than 12 months postoperatively. Eight patients (1-8) had follow-up examinations at 1 year or longer after injection. These patients show improvement across all measured voice parameters. Moreover, in 16 of the 20 patients, the procedure was performed at least 1

\section{V-RQOL Total Score}

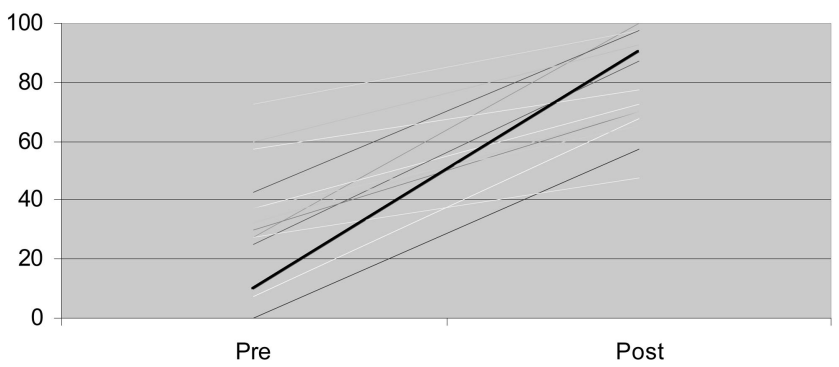

Fig. 4. Self-rated V-RQOL values before and after the injection laryngoplasty. year after onset of the vocal fold paralysis. Given the length of time that these patients had suffered with symptomatic vocal fold paralysis before presentation, any further benefit that could be accrued to spontaneous recovery seems unlikely. Therefore, it is reasonable to attribute these long-term voice results to the persistent benefit of Cymetra injection, which is the only intervention that they had received in this treatment period.

Although these long-term results remain anecdotal and limited to that portion of the study population that meets criteria for both duration of untreated paralysis and duration of follow-up, it is important to realize that the current study is the first to document such long-term benefit. Other studies, however, have suggested that such longer-term benefit might exist. For instance, Pearl et al. ${ }^{11}$ found persistent voice benefit in 7 of $8(87.5 \%)$ patients at 3 months posttreatment, but longer-term follow-up is not yet reported. Similarly, Lundy et al. ${ }^{16}$ do not examine any data longer than 1 month, although they do find significant voice benefit at that early time point and note that only two of their eight (25\%) patients required re-injection. In contrast with these results, Karpenko et al. ${ }^{4}$ report 1 month data for 10 patients and 3 month data for 7 patients and find no voice improvement at any time point, let alone any sustained benefit.

The different results in each of these studies might be attributable to several factors. For one, different injection 
techniques might lead to different resorption rates and therefore different results. It is interesting to note that the current study used suspension laryngoscopy and transoral injection using a laryngeal needle with a 27 gauge tip, whereas Pearl et al. ${ }^{11}$ and Karpenko et al. ${ }^{4}$ used similar transoral techniques with a 22-gauge and 21-gauge needle, respectively. In each of these studies, injections were performed at the mid-cord level into the thyroarytenoid muscle and lateral to the vocal process, into the vocal ligament. Lundy et al. ${ }^{16}$ injected into similar areas of the vocal fold, but they used a transcricothyroid technique in awake patients using a 25-gauge needle. Comparisons between studies are also difficult because of methodology artifact; when comparing this study with previous studies of Cymetra, it is important to realize that voice and videostroboscopic outcomes were assessed differently. There is not a uniform measure for judging the success or failure of treatment. Last, patient factors might also influence the results of injection. Although current studies do not permit elucidation of which factors might be predictive of increased benefit or decreased resorption, each study demonstrates variability within its own patient population. Perhaps different patterns of vocal use, immune response, or even phagocytosis can explain different results in different patients.

In examining those factors, which make comparison among different studies difficult, it should be noted that there are also potential limitations to the current study. For one, total sample size $(\mathrm{n}=20)$ is not very large, particularly at the data points with longer-term follow-up. Still, this study remains the largest current case series that has evaluated Cymetra injection laryngoplasty, and it is almost double the size of previous studies. Also, the methodology in this study for those patients injected before January 2002 is subject to recall bias because those patients were asked to provide preoperative self-ratings of voice and V-RQOL, and also to assess voice changes after injections in a retrospective fashion. Fortunately, this caveat applies only to patients 1 to 3 ; the remaining 17 patients provided self-voice ratings and V-RQOL at the time of presentation. Last, the nature of this study was to compare the most recently available follow-up data with the corresponding preoperative data. Although this technique allows for evaluation of outcomes at relatively long posttreatment time points, especially as compared with other studies, this technique does make it difficult to generalize concerning the time course of benefit and possible resorption in any rigorous fashion. However, the authors of this study feel that the results shown here are encouraging enough about the possible long-term benefits of Cymetra that a longer-term prospective study, which measures patient outcomes at several predetermined time points, appears warranted.

\section{CONCLUSION}

Overall, Cymetra injection laryngoplasty appears to have good vocal and videostroboscopic outcomes when comparing pretreatment data with corresponding posttreatment data in patients with unilateral vocal cord paralysis. In individual cases, improvement can be demon- strated to persist for greater than 12 months, suggesting that these benefits might be sustainable long term. If indeed Cymetra is found to offer long-term improvement, it may become the material of choice for injection laryngoplasty insofar as it offers the best combination of vocal benefit, length of outcome, biocompatability, easy handling, and lack of donor site morbidity. On the basis of the available retrospective data in this study, which is the first to suggest such a long-term benefit, we feel that further investigation into the role of Cymetra is warranted.

\section{BIBLIOGRAPHY}

1. Benninger MS, Ahuja AS, Gardner G, Grywalski C. Assessingoutcomes for dysphonic patients. $J$ Voice 1998;12: 540-550.

2. Brunings J. Uber eine neue behandlungsmethode der rekurranslahmung. Verhandl Ver Dtsch Laryngol 1911;18: 93-151.

3. Arnold GE. Vocal fold rehabilitation of paralytic dysphonia. IX. Technique of intracordal injection. Arch Otolaryngol 1962;76:358-368.

4. Karpenko AN, Dworkin JP, Meleca RJ, Stachler RJ. Cymetra injection for unilateral vocal cord paralysis. Ann Otol Rhinol Laryngol 2003;112:927-934.

5. Lewy RB. Experience with vocal cord injection. Ann Otol Rhinol Laryngol 1976;85:440-450.

6. Lewy RB. Teflon injection of the vocal cord: complications, errors, and precautions. Ann Otol Rhinol Laryngol 1983; 92:473-474.

7. Schramm VL, May M, Lavorato AS. Gelfoam paste injection for vocal cord paralysis: temporary rehabilitation of glottic incompetence. Laryngoscope 1978;88:1268-1273.

8. Brandenburg JH, Kirkham W, Koschkee D. Vocal cord augmentation with autogenous fat. Laryngoscope 1992;102: 495-500.

9. McCulloch TM, Andrews BT, Hoffman HT, et al. Long-term follow-up of fat injection laryngoplasty for unilateral vocal cord paralysis. Laryngoscope 2002;112:1235-1238.

10. Remacle M, Lawson G, Delos M, Jamart J. Correcting vocal fold immobility by autologous collagen injection for voice rehabilitation: a short-term study. Ann Otol Rhinol Laryngol 1999;108:788-793.

11. Pearl AW, Woo P, Ostrowski R, et al. A preliminary report on micronized Alloderm injection laryngoplasty. Laryngoscope 2002;112:990-996.

12. Sclafani AP, Romo T, Jacono AA, et al. Evaluation of acellular dermal graft in sheet (AlloDerm) and injectable (micronized AlloDerm) forms for soft tissue augmentation. Arch Facial Plast Surg 2000;2:130-136.

13. Livesey SA, Herndon DN, Hollyoak MA, et al. Transplanted acellular allograft dermal matrix. Potential as a template for the reconstruction of viable dermis. Transplantation 1995;60:1-9.

14. Jones FR, Schwartz BM, Silverstein P. Use of a nonimmunogenic acellular dermal allograft for soft tissue augmentation: a preliminary report. Aesthetic Surg $Q$ 1996;16: 196-201.

15. Hogikyan ND, Sethuraman G. Validation of an instrument to measure voice-related quality of life (V-RQOL). $J$ Voice 1999;13:557-569.

16. Lundy DS, Casiano RR, McClinton ME, Xue JW. Early results of transcutaneous injection laryngoplasty with micronized acellular dermis versus type-I thyroplasty for glottic incompetence dysphonia due to unilateral vocal fold paralysis. J Voice 2003;17:589-595.

17. Zapanta PE, Bielamowicz SA. Laryngeal abscess after injection laryngoplasty with micronized AlloDerm. Laryngoscope 2004;114:1522-1524. 